\title{
Melatonin for Pre- and Postoperative Pain and Anxiety: A Cancelled Clinical Trial
}

\section{Mikkel Zola Andersen, Bennedikte Kollerup Madsen, Dennis Zetner, Camilla Christophersen, Sofie Skovbo Jensen, Jacob Rosenberg}

Center for Perioperative Optimization, Department of Surgery, Herlev and Gentofte Hospitals, University of Copenhagen, Herlev, Denmark

Email: mikkelzola@gmail.com

How to cite this paper: Andersen, M.Z. Madsen, B.K., Zetner, D., Christophersen, C., Jensen, S.S. and Rosenberg, J. (2021) Melatonin for Pre- and Postoperative Pain and Anxiety: A Cancelled Clinical Trial. International Journal of Clinical Medicine, 12, 484-493.

https://doi.org/10.4236/ijcm.2021.1211044

Received: October 9, 2021

Accepted: November 22, 2021

Published: November 25, 2021

Copyright $\odot 2021$ by author(s) and Scientific Research Publishing Inc. This work is licensed under the Creative Commons Attribution International License (CC BY 4.0).

http://creativecommons.org/licenses/by/4.0/

\begin{abstract}
Designing and completing clinical intervention trials can be challenging. Many aspects must be considered to ensure that patients who fulfill the inclusion criteria for the intervention are identified and recruited effectively. The aim of this paper was to disseminate the results of a cancelled trial and present unpredictable barriers met underway, so future researchers can learn from these. The trial examined perioperative analgesic and anxiolytic effects of melatonin. It was registered at https://clinicaltrials.gov/ (NCT02386319) and a study protocol was published a priori. Participants were recruited from the plastic surgery ward of a Danish private hospital. The intended sample size of the trial was 72 patients based on power calculations of the outcome measures. During the six-month recruitment period, six patients were included, with only three completing the trial. Unpredictable barriers were poor communication between investigators and facility staff, lack of access to booking and operation schedules at the recruitment facility, the patient group being unwilling to participate, and the timing of recruitment conversations being unsuited as patients often did not have time to talk to the investigators. Too few data were collected to make any meaningful statistical analyses. Our trial was cancelled prematurely because of unpredictable barriers after commencing recruitment. Considering these barriers when designing a clinical trial may help future researchers avoid cancelling trials. Transparency of research is important and even prematurely cancelled trials should publish their findings.
\end{abstract}

\section{Keywords}

Randomized Controlled Trials, Barriers, Recruitment, Willingness to Participate, Inclusion 


\section{Introduction}

Randomized controlled trials are essential for researching effectiveness of medical treatments [1]. However, designing and completing these may include considerable barriers such as ethical, financial, and personnel issues [2]. Especially the recruitment phase has proven challenging for many trials [2] [3] [4]. Part of this may be explained by rigid legislation when conducting trials with patient involvement. For example, many countries must follow the Good Clinical Practice (GCP) guidelines, which ensure ethical and scientific quality of clinical trials by laying a set of rules to protect the rights of human research subjects [5] [6]. However, GCP has previously been criticized for increasing cost, complexity, and duration of research unnecessarily [7] [8].

Melatonin is an endogenous hormone that plays a large part in controlling the circadian rhythm and is used in many countries to treat sleep disturbances. Additionally, when given exogenously, melatonin has documented effects on pain and anxiety [9] [10]. Therefore, we planned to examine the effect of melatonin on perioperative pain and anxiety. In 2020 the clinical trial was cancelled due to several unforeseeable barriers. Therefore, the aim of this paper was to disseminate the few data collected in the trial and reflect upon barriers that resulted in early termination of the trial, so future researchers hopefully can succeed better than we did.

\section{Methods}

We conducted a randomized, double-blinded, placebo-controlled clinical trial measuring the effect of melatonin on pre- and postoperative pain and anxiety in patients undergoing elective breast augmentation. It was registered at clinicaltrials.gov (NCT02386319), and a protocol was published a priori [11]. For detailed methods of the trial, see this protocol. The first part of this study, examining pharmacokinetics of oral melatonin, was published in 2016 [12].

Patients were recruited after an outpatient visit with a plastic surgeon to determine eligibility for surgery. To be eligible for the trial, patients had to be eligible for primary breast augmentation or replacement of existing implants. Patients should take one tablet of $10 \mathrm{mg}$ melatonin or placebo four times between the evening before the operation and the evening of the operation. Patients were surveyed several times regarding pain, anxiety, and sleep between the morning of the surgery and 24 hours postoperatively. The intended sample size of the trial was 72 patients based on power calculations of the outcome measures. The trial was conducted in cooperation with a Danish private hospital, and all patients for the trial were recruited from the abovementioned hospital's plastic surgery ward.

All required ethical approvals for the trial were obtained before commencing. These include the Capital Region's Committee on Health Research Ethics (protocol number: H-8-2014-016), the Danish Data Protection Agency (journal number: VD-2019-101), and the Danish Medicines Agency (EudraCT number: 2014003789-25). The trial was registered at https://clinicaltrials.gov/ (NCT02386319) 
before commencing. Informed consent from study participants was obtained both written and verbally.

\section{Results}

Despite rather massive manpower, only six patients were included between December 2019 and May 2020, of which two completed the trial as intended (Figure 1). In total, 34 patients were screened for inclusion. Of the 28 that were not included in the trial, half $(13 / 28)$ either did not have time to hear about the trial or left the hospital before researchers could contact them after their outpatient visit. Six patients did not wish to participate after hearing about the trial. Five patients agreed to contact the investigators after considerations. Three of these

\section{Enrollment}

Assessed for eligibility $(n=34)$

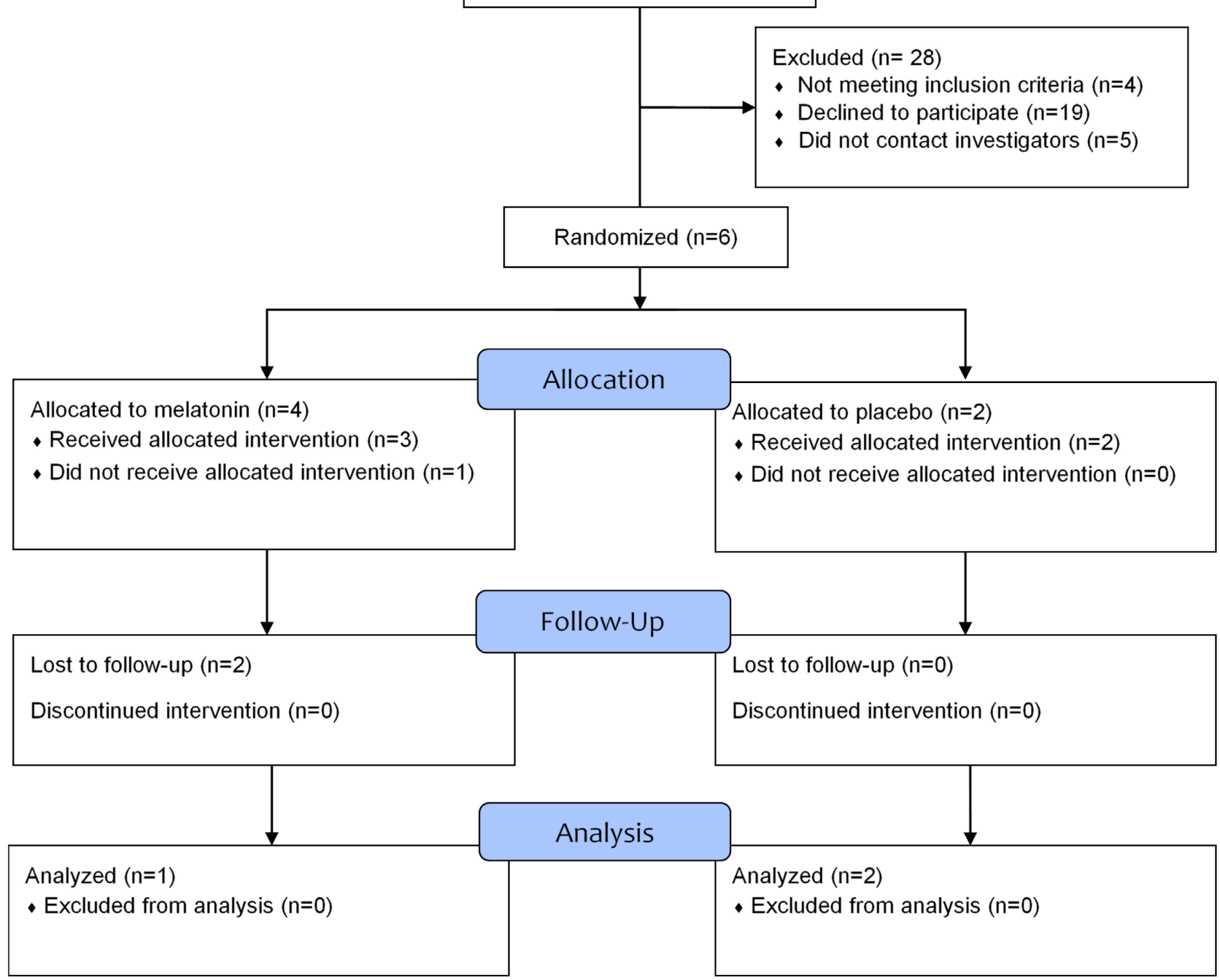

Figure 1. Flow chart of inclusion of patients. One patient did not receive the intervention because she was operated on another day than planned without the investigators' knowledge. Two patients were lost to follow-up as they were operated before planned time, so they did not receive their survey in time. The one patient analyzed in the melatonin group consequently answered surveys 1 - 2 hours after intended. 
five wished to consider if they were to have the operation, while two wished to consider if they wanted to participate in the trial. None of the five patients contacted the investigators. Two patients decided against breast augmentation and thus did not meet the inclusion criterion, and two patients were excluded based on the exclusion criteria. Of the six included patients, three patients answered all surveys in the trial. However, one of these patients answered all the surveys 1 - 2 hours late, introducing considerable risk of bias to their results. Of the three patients that did not complete the trial, one underwent surgery on a different day without informing the research team, and the remaining two underwent surgery on the correct day but earlier than planned, again without informing the research team. This resulted in the patients not receiving their surveys on time. Thus, in total, two (6\%) of 34 screened patients completed the trial as intended.

We estimated 150 operations yearly based on information provided by the hospital. Despite this, we only encountered 34 patients in six months. This was partly due to poor communication with the ward staff, resulting in the investigators not being contacted when patients were re-booked or made an appointment on short notice, which could happen daily. With the rate of inclusion at the time of cancellation, the trial was set to last 10 years from the beginning of inclusion. Therefore, we chose to terminate the trial as this would prove too costly.

As major barriers for recruitment in our trial, we highlight the time of recruitment conversation, the recruitment demographic, and the recruitment facility (Figure 2). Firstly, patients were approached for inclusion following a non-obligating outpatient visit with a plastic surgeon to determine if the patient was a candidate for operation. Some declined to participate in the trial, as they did not know if they would have the surgery. Patients were not warned at the time of booking that researchers would contact them upon arrival at the hospital, as it was not logistically possible, and some did not have time to hear about the trial when approached by investigators. Many left the hospital despite being told at the time of arrival that the investigators would like to talk with them after the plastic surgeon. Secondly, the demographic of young females going for self-payed, cosmetic, one-day surgery in a country with otherwise free public healthcare proved challenging. Several eligible patients were not interested in participating, and others that agreed to contact the investigators did not do so. Thirdly, coming from another institution none of the investigators had any familiarity or connections with the staff when inclusion began, making cooperation more difficult. Additionally, the investigators did not have access to electronic health records in this hospital and to get permission would require several complex and time-consuming applications with ethical and data committees probably lasting up to a year. Therefore, the investigators could not see the actual bookings, which made regular staff involvement necessary. Furthermore, the trial's contact person from the ward was on unexpected leave throughout the inclusion period due to personal matters. This unlucky circumstance made 


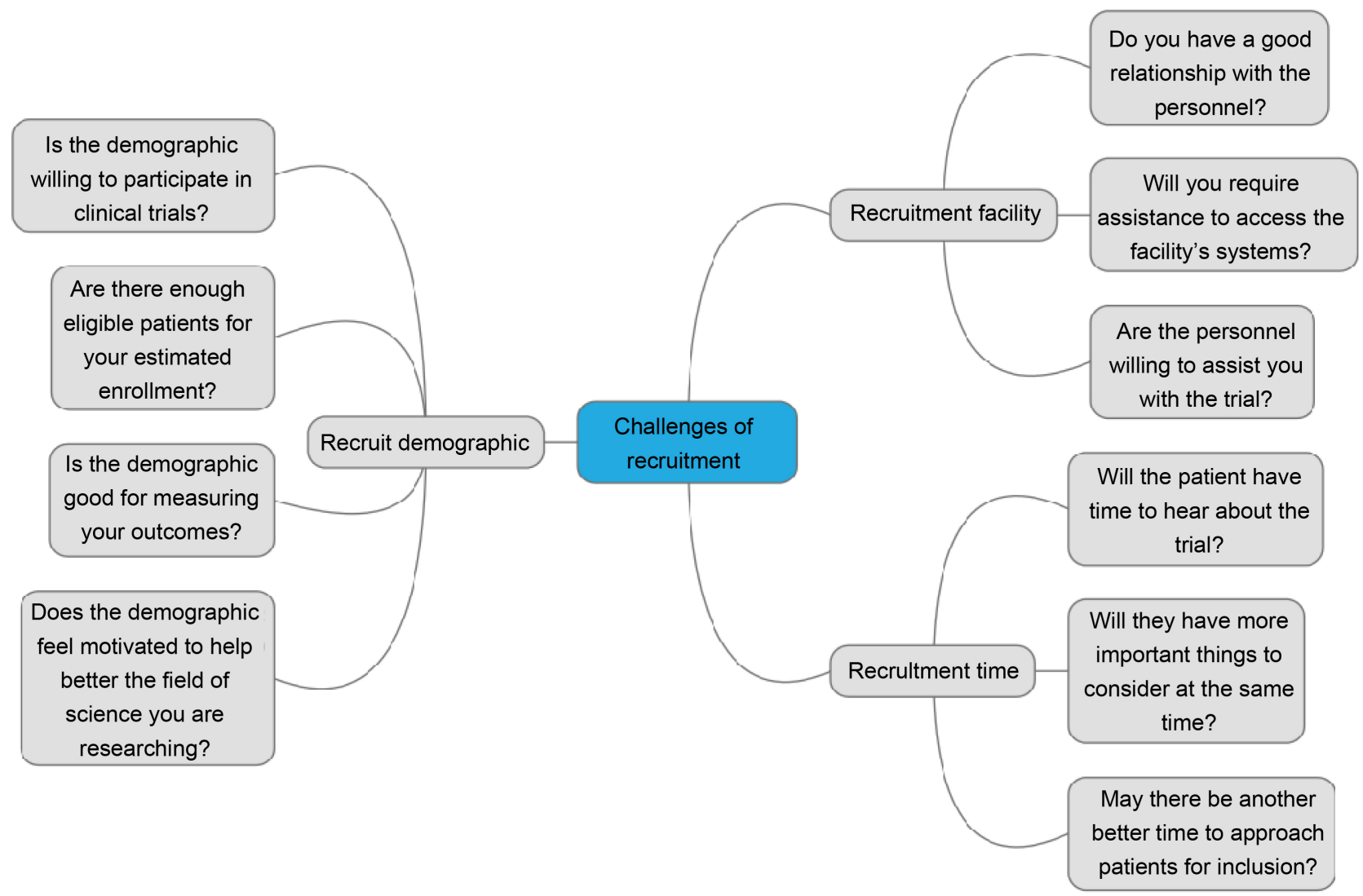

Figure 2. Barriers to consider before starting a randomized clinical trial.

communication at the hospital even more challenging.

The data collected from the six included patients are displayed in Table 1. These were too few to make any meaningful analyses but are reported to offer transparency.

This clinical trial was cancelled early owing to unpredictable barriers including: the timing of recruitment conversations being unsuited, as patients often did not have time to talk to the investigators; the patient group being unwilling to participate; poor communication between investigators and facility staff; and lack of access to electronic health records at the recruitment facility. The data collected were too few to make meaningful analyses.

During the six months of inclusion only six patients agreed to participate, of which two patients completed the trial as intended. With the rate of inclusion at the time of trial cancellation, inclusion was set to last 10 years. As randomized controlled trials are costly, and since many other factors around patient care may change in 10 years, it was not possible to keep the trial going for this long, thus, we decided to cancel the trial.

Regarding the time of recruitment conversation, it was not possible to recruit at a better time, as patients would not visit the hospital between attempted recruitment and the first dose of trial drug. Therefore, we had to give the patients the trial drug to bring home after inclusion. According to GCP regulations all 
Table 1. Results from the three patients that completed the trial. ID 5 did not take the study medicine and thus had no results to report.

\begin{tabular}{|c|c|c|c|c|c|}
\hline Characteristic & ID 1 & ID 2 & ID $3^{*}$ & ID $4^{* *}$ & ID $6^{*}$ \\
\hline Intervention & Placebo & Placebo & Melatonin & Melatonin & Melatonin \\
\hline Age & 22 & 25 & 21 & 52 & 51 \\
\hline \multicolumn{6}{|l|}{ Preoperative visit } \\
\hline VAS pain (rest) & 0 & 0 & 0 & 0 & 1 \\
\hline VAS pain (movement) & 0 & 0 & 0 & 0 & 0 \\
\hline VAS anxiety & 3 & 0 & 50 & 0 & 4 \\
\hline State anxiety & 35 & 25 & 32 & 27 & 24 \\
\hline Trait anxiety & 42 & 29 & 45 & 31 & 32 \\
\hline \multicolumn{6}{|l|}{ Morning of surgery } \\
\hline KSS & 3 & 7 & 3 & 1 & 3 \\
\hline Hours of sleep & 7 & 5.5 & 6.5 & 4 & 5 \\
\hline Quality of sleep & 25 & 27 & 11 & 18 & 70 \\
\hline Fatigue & 3 & 6 & 3 & 1 & 7 \\
\hline Wellbeing & 25 & 31 & 1 & 100 & 50 \\
\hline \multicolumn{6}{|l|}{$60 \mathrm{~min}$ before surgery } \\
\hline VAS anxiety & 21 & 18 & - & 2 & - \\
\hline State anxiety & 33 & 31 & - & 43 & - \\
\hline \multicolumn{6}{|l|}{ Intraoperative } \\
\hline Remifentanil usage $(\mu \mathrm{g})$ & 40 & 38 & - & 25 & - \\
\hline Propofol usage (mL) & 62 & 238 & - & 41 & - \\
\hline \multicolumn{6}{|l|}{1 hour postoperative } \\
\hline VAS pain (rest) & 56 & 62 & - & 23 & - \\
\hline VAS pain (movement) & 67 & 80 & - & 77 & - \\
\hline VAS anxiety & 13 & 0 & - & 0 & - \\
\hline \multicolumn{6}{|l|}{2 hours postoperative } \\
\hline VAS pain (rest) & 66 & 18 & - & 38 & - \\
\hline VAS pain (movement) & 77 & 36 & - & 77 & - \\
\hline VAS anxiety & 21 & 0 & - & 6 & - \\
\hline \multicolumn{6}{|l|}{4 hours postoperative } \\
\hline VAS pain (rest) & 72 & 8 & - & 0 & - \\
\hline VAS pain (movement) & 93 & 27 & - & 95 & - \\
\hline VAS anxiety & 32 & 0 & - & 0 & - \\
\hline State anxiety & 36 & 27 & - & 31 & - \\
\hline \multicolumn{6}{|l|}{6 hours postoperative } \\
\hline VAS pain (rest) & 35 & 11 & - & 18 & - \\
\hline VAS pain (movement) & 35 & 28 & - & 20 & - \\
\hline Opioid usage (oxycodon mg) & 10 & 10 & - & 17.5 & - \\
\hline
\end{tabular}




\section{Continued}

8 hours postoperative

$$
\begin{aligned}
& \text { VAS pain (rest) } \\
& \text { VAS pain (movement) }
\end{aligned}
$$

Time of release from hospital

Time in recovery (hours)

Total time in hospital (hours)

Morning after surgery

\section{KSS}

Hours of sleep

Quality of sleep

Fatigue

Wellbeing

24 hours postoperative

$$
\begin{aligned}
& \text { Opioid usage (mg) } \\
& \text { VAS pain (rest) } \\
& \text { VAS pain (movement) }
\end{aligned}
$$

$$
19
$$

6

16

1

$$
3
$$

$$
1.5
$$$$
7
$$

3
6
2

6

71

63

3

6.5

28

4

30

2.5

32

74

$\begin{array}{lll}40 & - & 45 \\ 15 & - & 0 \\ 30 & - & 30\end{array}$

VAS: Visual Analog Scale (1 - 100); STAI: State-Trait Anxiety Inventory. Scale from 20 - 80 each for state anxiety and trait anxiety, with higher values indicating more anxiety [13]. KSS: Karolinska Sleepiness Scale. Scale from 1 - 9 with higher values indicating more sleepiness [14]. Quality of sleep: Scale from 1 - 100 with higher values indicating lower quality of sleep. Fatigue: Scale from 1 10 with higher values indicating more fatigue. Wellbeing: Scale from $1-100$ with higher values indicating less wellbeing. *: Did not complete the trial; ${ }^{\star *}$ : Answered surveys 1 - 2 hours after intended.

aspects of trial drug storage should be documented sufficiently [6]. As the patients could not document storage of the trial drug in their home, we were unable to retrieve study medicine if the patient decided to drop out of the trial. This was despite the only storage requirement for the drug being storage below $25^{\circ} \mathrm{C}$, which would be easy for the patients to comply with, even when storing the medicine in their homes. As we did not have much more medicine than needed to meet power calculations, losing medicine to patients dropping out was costly. If this GCP regulation did not exist it would be more manageable if patients dropped out, as we could retrieve the medicine if the patient had not taken the first dosage. An operation could also be chosen that required a follow-up visit to the hospital after booking the operation and use this time for inclusion, e.g. an appointment with an anesthesiologist prior to surgery. This might result in patients being more decided on operation at the time of recruitment conversation. Alternatively, an operation where patients are very certain, prior to their appointment with the doctor, that they will indeed have the surgery could be chosen, e.g. elective inguinal hernia repair. Attempts to inform the patient that they would be asked by an investigator to participate in a clinical trial at the time of outpatient visit booking may have improved inclusion rates. In general, howev- 
er, it is uncertain which information must be presented to patients to improve recruitment [15].

The operation chosen resulted in the demographic for inclusion and while the demographic was good for measuring the outcomes, willingness to participate and compliance was not optimal. It has been argued that recruitment of surgical patients for clinical trials is unpredictable [16]. Patients paying for their own operation in a country with free public health care may be less willing to participate in clinical trials, if this group of patients does not feel obligated to contribute to research, as they have paid for their own treatment and are not offered anything in return. Monetary incentives have been shown to be successful in recruiting and maintaining patients in clinical trials [15] [17] [18] [19] [20] [21]. However, in Denmark, where this trial was conducted, monetary incentives for patients are not allowed. Previous trials have had difficulties recruiting patients to trials of self-payment versus funded treatment [22]. This may be interesting to examine qualitatively in the future. Thus, another type of surgery, perhaps without patient payment involved, should probably have been chosen.

Regarding the recruitment facility, it is important to ensure a good relationship with the ward staff to ease communication. In retrospect, as we were not contacted with updates of patient bookings, we should have contacted the staff more often for information. However, it is also important not to annoy the staff with constant contact. If possible, using a hospital where one of the investigators works regularly might improve communication and recruitment. Yet, for our trial, the operation chosen did not allow this. We had not acquired permission to access the electronic health records at the hospital before starting inclusion. This should be a consideration when planning a trial. Had we done so, it would have allowed the investigators to follow up on patient bookings and operations without involving the staff. Access to electronic systems may potentially make clinical research more effective [23], but ethical issues regarding security must also be considered [24]. Alternatively, involving the staff at the hospital in the recruitment process to a higher degree may improve communication and recruitment, but this may be difficult when there are no incentives involved.

\section{Conclusion}

Many barriers may arise in designing and conducting clinical trials, and some of these may present themselves late in the process. Our trial was cancelled due to unpredictable barriers during the recruitment phase of the trial. Major barriers were related to poor communication between investigators and ward staff, lack of access to electronic health records, the patient group being unwilling to participate, and the recruitment time being unsuited as patients often did not have time to talk to the investigators. These barriers should be considered by future researchers before commencing inclusion. Transparency in research is important, and results of clinical trials should be reported, even if they do not offer sound conclusions. Our trial is one such example. 


\section{Conflicts of Interest}

The authors declare no conflicts of interest regarding the publication of this paper.

\section{References}

[1] Hariton, E. and Locascio, J.J. (2018) Randomised Controlled Trials-The Gold Standard for Effectiveness Research. BJOG, 125, 1716. https://doi.org/10.1111/1471-0528.15199

[2] Varse, F., Janani, L., Moradi, Y., Solaymani-Dodaran, M., Baradaran, H.R. and Rimaz, S. (2019) Challenges in the Design, Conduct, Analysis, and Reporting in Randomized Clinical Trial Studies: A Systematic Review. Medical Journal of the Islamic Republic of Iran, 33, 37. https://doi.org/10.47176/mjiri.33.37

[3] McDonald, A.M., Knight, R.C., Campbell, M.K., Entwistle, V.A., Grant, A.M., Cook, J.A., et al. (2009) What Influences Recruitment to Randomised Controlled Trials? A Review of Trials Funded by Two UK Funding Agencies. Trials, 7, 9. https://doi.org/10.1186/1745-6215-7-9

[4] Thoma, A., Farrokhyar, F., McKnight, L. and Bhandari, M. (2010) Practical Tips for Surgical Research: How to Optimize Patient Recruitment. Canadian Journal of Surgery, 53, 205-210.

[5] Vijayananthan, A. and Nawawi, O. (2008) The Importance of Good Clinical Practice Guidelines and Its Role in Clinical Trials. The Biomedical Imaging and Intervention Journal, 4, e5. https://doi.org/10.2349/biij.4.1.e5

[6] Guideline for Good Clinical Practice E6(R2). https://www.ema.europa.eu/en/documents/scientific-guideline/ich-e-6-r2-guideline -good-clinical-practice-step-5_en.pdf

[7] Grimes, D.A., Hubacher, D., Nanda, K., Schulz, K.F., Moher, D. and Altman, D.G. (2005) The Good Clinical Practice Guideline: A Bronze Standard for Clinical Research. The Lancet, 366, 172-174. https://doi.org/10.1016/S0140-6736(05)66875-4

[8] Mentz, R.J., Hernandez, A.F., Berdan, L.G., Rorick, T., O’Brien, E.C., Ibarra, J.C., et al. (2016) Good Clinical Practice Guidance and Pragmatic Clinical Trials: Balancing the Best of Both Worlds. Circulation, 133, 872-880. https://doi.org/10.1161/CIRCULATIONAHA.115.019902

[9] Srinivasan, V., Pandi-Perumal, S.R., Spence, D.W., Moscovitch, A., Trakht, I., Brown, G.M., et al. (2010) Potential Use of Melatonergic Drugs in Analgesia: Mechanisms of Action. Brain Research Bulletin, 81, 362-371. https://doi.org/10.1016/j.brainresbull.2009.12.001

[10] Ochoa-Sanchez, R., Rainer, Q., Comai, S., Spadoni, G., Bedini, A., Rivara, S., et al. (2012) Anxiolytic Effects of the Melatonin MT2 Receptor Partial Agonist UCM765: Comparison with Melatonin and Diazepam. Progress in Neuro-Psychopharmacology \& Biological Psychiatry, 39, 318-325. https://doi.org/10.1016/j.pnpbp.2012.07.003

[11] Kollerup Madsen, B., Zetner, D. andersen, L.P.K. and Rosenberg, J. (2019) The Anxiolytic and Analgesic Effects of Melatonin: A Study Protocol for a Randomized, Double-Blind, Placebo-Controlled Study. Melatonin Research, 2, 22-34.

https://doi.org/10.32794/mr11250019

[12] Harpsøe, N.G. andersen, L.P.K., Mielke, L.V., Jønsson, B., Jenstrup, M.T., Gögenur, I., et al. (2016) Pharmacokinetics of Repeated Melatonin Drug Administrations Prior to and after Surgery. Clinical Drug Investigation, 36, 1045-1050. 
https://doi.org/10.1007/s40261-016-0452-5

[13] Spielberger, C.D., Gorsuch, R.L., Lushene, R., Vagg, P.R. and Jacobs, G.A. (1983) Manual for the State-Trait Anxiety Inventory. Consulting Psychologists Press, Palo Alto.

[14] Kaida, K., Takahashi, M., Åkerstedt, T., Nakata, A., Otsuka, Y., Haratani, T., et al. (2006) Validation of the Karolinska Sleepiness Scale against Performance and EEG Variables. Clinical Neurophysiology, 117, 1574-1581. https://doi.org/10.1016/j.clinph.2006.03.011

[15] Treweek, S., Pitkethly, M., Cook, J., Fraser, C., Mitchell, E., Sullivan, F., et al. (2018) Strategies to Improve Recruitment to Randomised Trials. Cochrane Database of Systematic Reviews, 2, MR000013. https://doi.org/10.1002/14651858.MR000013.pub6

[16] Jack, W.J.L., Chetty, U. and Rodger, A. (1990) Recruitment to a Prospective Breast Conservation Trial: Why Are So Few Patients Randomised? BMJ, 301, 83-85. https://doi.org/10.1136/bmj.301.6743.83

[17] Yu, S., Alper, H.E., Nguyen, A.M., Brackbill, R.M., Turner, L., Walker, D.J., et al. (2017) The Effectiveness of a Monetary Incentive Offer on Survey Response Rates and Response Completeness in a Longitudinal Study. BMC Medical Research Methodology, 17, 77. https://doi.org/10.1186/s12874-017-0353-1

[18] Griffin, J.M., Simon, A.B., Hulbert, E., Stevenson, J., Grill, J.P., Noorbaloochi, S., et al. (2011) A Comparison of Small Monetary Incentives to Convert Survey NonRespondents: A Randomized Control Trial. BMC Medical Research Methodology, 11, 81. https://doi.org/10.1186/1471-2288-11-81

[19] Agarwal, A., Raad, D., Kairouz, V., Fudyma, J., Curtis, A.B., Schünemann, H.J., et al. (2016) The Effect of a Monetary Incentive for Administrative Assistants on the Survey Response Rate: A Randomized Controlled Trial. BMC Medical Research Methodology, 16, 94. https://doi.org/10.1186/s12874-016-0201-8

[20] Pit, S.W., Vo, T. and Pyakurel, S. (2014) The Effectiveness of Recruitment Strategies on General Practitioner's Survey Response Rates-A Systematic Review. BMC Medical Research Methodology, 14, 76. https://doi.org/10.1186/1471-2288-14-76

[21] Hardy, P., Bell, J.L., Brocklehurst, P., Bick, D., Briley, A., Juszczak, E., et al. (2016) Evaluation of the Effects of an Offer of a Monetary Incentive on the Rate of Questionnaire Return during Follow-Up of a Clinical Trial: A Randomised Study within a Trial. BMC Medical Research Methodology, 16, 82. https://doi.org/10.1186/s12874-016-0180-9

[22] Tudor, K., Jebb, S.A., Manoharan, I. and Aveyard, P. (2020) Brief Interventions for Obesity When Patients Are Asked to Pay for Weight Loss Treatment: An Observational Study in Primary Care with an Embedded Randomised Trial. British Journal of General Practice, 70, e348-e355. https://doi.org/10.3399/bjgp20X708797

[23] Coorevits, P., Sundgren, M., Klein, G.O., Bahr, A., Claerhout, B., Daniel, C., et al. (2013) Electronic Health Records: New Opportunities for Clinical Research. Journal of Internal Medicine, 274, 547-560. https://doi.org/10.1111/joim.12119

[24] Powell, J. and Buchan, I. (2005) Electronic Health Records Should Support Clinical Research. Journal of Medical Internet Research, 7, e4.

https://doi.org/10.2196/jmir.7.1.e4 